\title{
Facilitating Personalisation in Epilepsy with an IoT Approach
}

\author{
S.A McHale, E.Pereira \\ Department of Computer Science \\ Edge Hill University \\ Ormskirk, UK
}

\begin{abstract}
The premises made in this paper put the future of personalisation in epilepsy into focus, a focus that shifts from a one-size fits all to a focus on the core of the epilepsy patients' individual characteristics. The emerging approach of personalised healthcare is known to be facilitated by the Internet of Things (IoT) and sensor-based IoT devices are in popular demand for healthcare providers due to the constant need for patient monitoring. In epilepsy, the most common and complex patients to deal with correspond to those with multiple strands of epilepsy. These extremely varied kind of patients should be monitored precisely according to their identified key symptoms and specific characteristics then treatment tailored accordingly. Consequently, paradigms are needed to personalise this information. By focusing upon personalised parameters that make epilepsy patients distinct this paper proposes an IoT based Epilepsy monitoring model endorsing a more accurate and refined way of remotely monitoring the 'individual' patient.
\end{abstract}

Keywords-IoT; healthcare systems; smart healthcare; personalisation

\section{INTRODUCTION}

By integrating IoT sensor-based devices deployed remotely and personalised patient data into a combined monitoring framework a vision of personalisation is realised. This study revealed some irrefutable evidence derived from patient profile analysis and experimental data that seizure detection using sensors positioned on different parts of a patents body ultimately makes an impact on the monitoring of epilepsy, endorsing that modern computer science is providing a timely chance for a more personalised approach to the monitoring and management of epilepsy.

The chances of capturing seizure data can be greatly increased if a correctly assigned sensor is placed on the correct part of the patient's body and ultimately, such a concept could "enhance the overall monitoring scheme of a patient usually performed by caring persons, who might occasionally miss an epileptic event' [1].

This paper is organised as follows. In section II and section III the state of the art is analysed; the complexity of epilepsy together with smart healthcare monitoring approaches are highlighted. There is also a focus upon the sensors available for epilepsy following on with an emphasis on the limitations for a personalised approach. Section IV presents the driving questions in this study and describes the experiment and findings from capturing seizure data. Section V introduces the proposed IoT based Epilepsy monitoring model and reveals the
PMP (Personalised Monitoring Plan) framework whereby the patient can be matched with the correct device, while Section VI presents how this was evaluated and Section VII outlines long term use. Concluding remarks are drawn in Section VIII.

\section{Motivation}

Epileptic seizure monitoring and management is challenging. Most current studies of epileptic seizure detection disclose drug resistant epilepsy still lacks an ultimate solution, despite the increase in anti-epileptic drugs [2].

Epilepsy is not a single disease, but a family of syndromes that share the feature of recurring seizures. In some instances, it may be related to a genetic aetiology, or it can occur in association with metabolic disorders, structural abnormalities, infection or brain injury [3].

In the United Kingdom epilepsy affects 3 million people and in the United States it is the 4th most common neurologic disorder, only migraine, stroke, and Alzheimer's disease occurs more frequently [4]. There are around 60 different types of seizure and a person may have more than one type. Seizures vary depending on where in the brain they are happening. Some people remain aware throughout, while others can lose consciousness [5].

Aside from their unpredictability, the worst part of having seizures is their utter complexity. The complex nature of epilepsy is noticeable in the variation of seizures types and symptoms between one patient and another. Distinguishing or classifying an individual epilepsy patient makes it difficult to manage and monitor. The negative impact of uncontrolled seizures spreads beyond the individual to affect their family, friends, and society. Chronic anxiety is experienced by the families and friends of people with epilepsy and many lives are adjusted to ensure the safety of their loved one. Novel approaches to epilepsy treatment are still greatly needed [6] novel therapies that better manage and monitor seizures as well as technology can help to handle the consequences of seizures.

Insufficient knowledge about epilepsy, which is a very common disorder, has a great and negative impact on people with epilepsy, their families and communities, and the healthcare systems. There is need for a better understanding of the disease to make way for new approaches to monitor it.

In the modern day of personalised medicine and rapid advancements in IoT a question that needs addressing is whether epilepsy monitoring can benefit from personalised 
approach. Can the IoT have the potential to significantly improve the 'patients' daily lives whose seizures cannot be controlled by either drugs or surgery [7]?

\section{A. Smart Healthcare Monitoring Approaches}

In the history of time it is only relatively recently that computers began to assist healthcare monitoring, in 1950s' patients began to be continuously monitored by computerised machines [8] and clinical monitoring was first envisaged in the home [9]. For computer assistance to epilepsy it was not until 1972 in the field of imaging, when computerized tomography (CT) was invented by the British engineer Godfrey Hounsfield [10] and only in recent decades where specific epilepsy healthcare 'monitoring systems' have been proposed.

Much of this recent growth being due to the advent of current IoT technology whereby the rise of 'smart environment' approaches to healthcare monitoring is witnessed. There are many IoT approaches for the monitoring and management of epilepsy many of which encompass a network of connected smart devices which are equipped with sensors either embedded in clothing or smart phones, to either detect, predict or manage epilepsy. Discoveries disclose how IoT is utilised to support the ever-growing trend of personalised healthcare. These recent 'smart' approaches in healthcare demonstrate the trend toward 'sensor use' and 'remote monitoring'.

\section{RELATED WORK}

Researchers are bounding toward the new generation of smart technology and IoT (Internet of Things). Novel devices such as smart watches, smart bands \& smart clothing are all competing for the ultimate solution. Yet it is found there is limited research which focuses upon the concept of a more holistic, personalised approach to help manage epilepsy.

One study deemed the significance of attention on smart technologies and its potential to identify early indicators of cognitive and physical illness [11] and observed that researchers have argued and predicted that assessing individuals in their 'everyday environment' will provide the most 'valid' information about everyday functional status [12].

Indeed, there is evidence recently of this indication as several IoT platforms to manage \& monitor healthcare remotely, are observed. For example, one IoT paradigm comprising of Wireless Health Sensors (WHS) permits the continuous monitoring of biometric parameters such as pulse rate, pulmonary functional quality, blood pressure and body temperature [13]. This IoT paradigm is being used to assist predictive analysis via smart healthcare systems by a medical practitioner. Using sensors connected to Arduino patient status is tracked, and by a Wi-fi connection data is collected and transmitted and can receive user requests. This data is shared with doctors through a website where the doctor can analyse the condition of the patient and provide further details online and intimate patient about future severity well in time [13].

\section{A. Sensors for Epilepsy}

EEG, an electroencephalogram is a recording of brain activity. This is the chief gold standard method used within hospitals to detect and monitor seizures. Several approaches have been reported with the aim to embed this method in other settings and platforms. Developments in some topics have been published, such as modelling the recorded signals [14] [15] or the design of portable EEG devices to deploy such models.

As an alternative and sometimes supplement to EEG there exist many sensors embedded in clothing or worn on the body to obtain bio-signals such as gyroscopes, accelerometers, pulse rate, temperature sensors, magnetometers, galvanic skin response sensors (GSR), implanted advisory system, electromyography, video detection systems, mattress sensor, and audio systems [16].

A large amount of apps have been published more recently especially in the commercial sector for the detection and management of seizures using either the Smartphone sensors or external sensors, for example Epdetec [17] and Myepipal [18] and web logging which facilitates the way a patient records daily information concerning her/his epileptic events, medication, and news, My Epilepsy Diary [19] and Epidiary [20]. Another app attracting attention and recently reviewed in the press is the Alert App by Empatica. This app sends caregivers an automated SMS and phone call when it detects unusual patterns that may be associated to a convulsive seizure [21] yet it is only designed to work with the Embrace Smartband by Empatica and can prove expensive for the user [22].

Regrettably, there are few specific sensor detection options for each specific seizure type, this is an imminent requirement for patients and their carers. Ideally when choosing a seizure detection device, the patient-specific seizure semiology's should be considered [16]. Thus, highlighting the need for a type of monitoring that distinguishes one patient from another and depicting the need for devices to pinpoint the patientspecific signs and symptoms.

\section{B. Addressing the Gaps}

Despite the focus in literature on smart healthcare monitoring approaches there is limited emphasis on the embracing of a truly personalised approach for epilepsy as previously described. Even though the 'diversity' of epilepsy is acknowledged and has been identified in other studies, i.e. by highlighting the importance of distinguishing each 'seizure type', there is still a gap to address such parameters. The 'seizure type' is just one of many parameters that can distinguish one seizure patient from another. Therefore, these very individual characteristics can be further identified to address the challenge to achieve a truly personalised approach to managing epilepsy.

More so recently it is recognised that devices should specially take into account the user's seizure types and personal preferences [23], focus should be shifting not only on the desires of the users but seizure detection devices should be able to 'adapt' to the patient's characteristics and seizures [23].

It is already becoming known that wearing sensors on the body is starting to be popular, as observed recently in a 2018 study where a great interest was highlighted in the use of wearable technology for epilepsy carers, this being independent of demographic and clinical factors and remarkably outpacing data security and technology usability 
concerns thus demonstrating the vital factor of comfortability [24]. Yet as discovered during a review to select the best sensor for each individual patient there was limited data on which was the best sensor for each seizure type, this was unfortunate despite an internationally active research effort, signifying the gap in knowledge, again, for understanding the individual epilepsy patient [16].

\section{EXPERIEMNT AND FINDINGS}

This section discusses the experiment that was performed to capture seizure data, obtained from sensors, which are positioned on different parts of the patient's body. This was done to test the assumption that it is 'the individual profile' that makes the difference in which device to choose. The results from this experiment are used to inform a typical model or a PMP (Personalised Monitoring Plan) discussed in the next section.

The actual 'sensor', and their 'position' (worn by the patient) are significant for epilepsy and the focus in the experiment was on how patients exhibit behaviour, rather than any actual testing of devices. It was therefore important to choose the most accurate sensors for monitoring epilepsy; those were found to be the accelerometer and heart-rate sensors, although latest studies suggest making use of other sensors too such as peripheral temperature, photo plethysmography (blood circulation), respiratory sensors [25], and galvanic (changes in sweat gland activity) among others [26].

\section{A. Preliminary Investigations}

Numerous studies have been previously been conducted with sensors and use for epilepsy [27] [28]. Since the 'gold standard' for epilepsy monitoring is video-EEG monitoring (which takes place within hospitals) [29] the driving questions addressed here were:

1) Can the patient be just as accurately monitored at home with an inexpensive, easily obtainable accelerometer and heart-rate sensor-based device?

2) Can the individual requirements of the patient be pinpointed? If so, is it possible that these sensors can be worn at home (a personalised approach) and be just as effective as using EEG monitoring in the hospital setting?

From the analysis of the patient data it is clear that a patient profile based on particular characteristics can indicate which position the sensor is best placed on the patient's body.

Sample patient profiles where selected based upon criteria informed from discussions with clinicians. For example, Patient Profile 1 seizures begins with the right arm suddenly raising, therefore can the sensor be placed upon the right shoulder? Patient Profile 4 has a lot of shaking during their Focal Onset Seizures with shaking starting on the left arm so therefore can the sensor be useful attached to the left wrist? Whereas Patient Profile 5 begins their seizures with severe tremors on the right leg, can the sensors detect movement and heart-rate changes with sensor in this position?

During the investigation practicable devices to use in the experiment to monitor epilepsy were analysed. The 'Fitbit
Ionic' was chosen as the best option since both the heart-rate and accelerometer can be extracted. The commercial activity device has been used in other studies, most notably recently whereby it used data from more than 47,000 Fitbit users in five U.S. states and data revealed that with Fitbit use the state-wide predictions of flu outbreaks were enhanced and accelerated [30]. This use demonstrates the viability and potential suitability of Fitbit as a healthcare device.

\section{B. Experimental Description}

The objectives of the experiment were to assess the movement from the accelerometer sensor and the pulse from the heart-rate sensor in the detection of epileptic seizures. Participants with confirmed epilepsy are recruited. The noninvasive wrist, leg, knee or arm-worn sensors are used to acquire heart-rate activity and movements. The study evaluated the movement from the accelerometer sensor and the pulse from the heart-rate sensor in the detection of an epileptic seizure. Over a period of 5 days the patients were asked to wear the device and continue recording seizures in their seizure diary. The study also evaluated any differences in result due to the 'position' of the sensor on the body together with the patients' acceptability \& comfort.

The instructions contained daily forms for the patient to complete, hence, keeping a diary of the times of seizure, if they did not use this method an EEG recording was obtained. This way the actual time stamp of the patients recorded seizure can be checked against the server time stamp observations of the seizure, so for example if the patient records their seizure at 10.20am and the server readings reveal heart-rate peaks and rapid movement from the accelerometer also at 10.20am, then this confirms the server readings match the patients (or EEG) known seizure occurrence, see Fig. 1, Seizure Time Stamps.

\section{Experimental Results}

The heart-rate and accelerometer sensors used to detect characteristics of seizure events can successfully record seizure data, without need for participant cooperation beyond wearing the sensor-based device, even recharging the battery (battery life is 5 days when fully charged) was not required by the participants. Both the sensors detected the 'shaking' seizures correctly as can be seen in observation d HP4, in Fig. 2, Observations, set 1.

The effectiveness was also verified by "Non-seizure times" which are more easily recognised in sleep due to inactivity demonstrating that the sensors worked properly: see the above random time periods, in Fig. 2 whereby seizures did not occur for HP4 in Observation ' $k$ ' and ' $\mathrm{i}$ '.

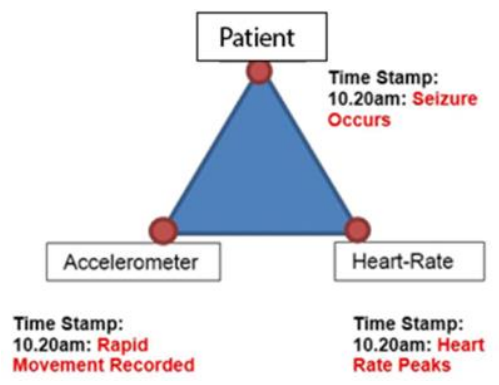

Fig. 1. Seizure Time Stamps. 
Observation d HP4
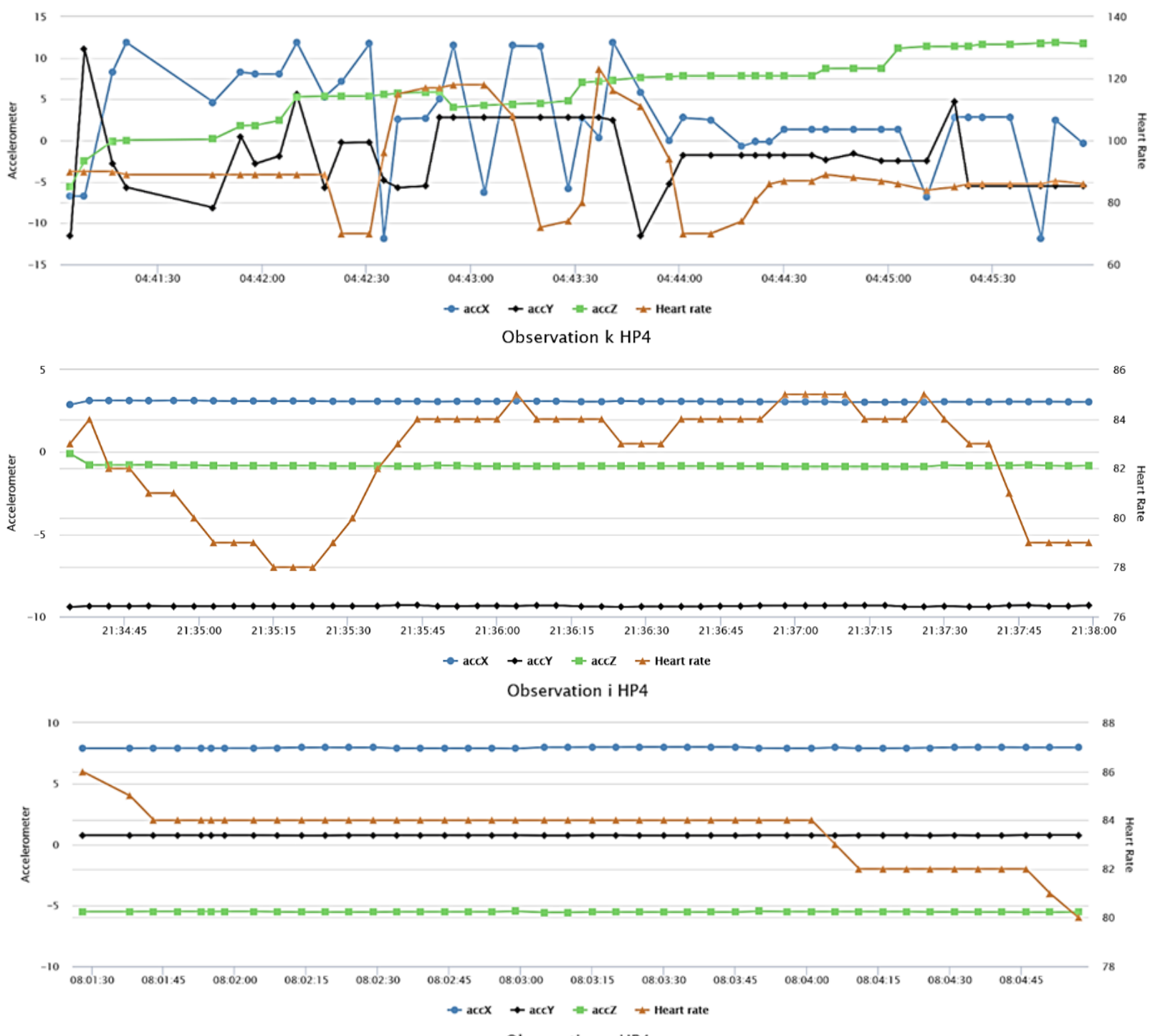

Observation c HP4

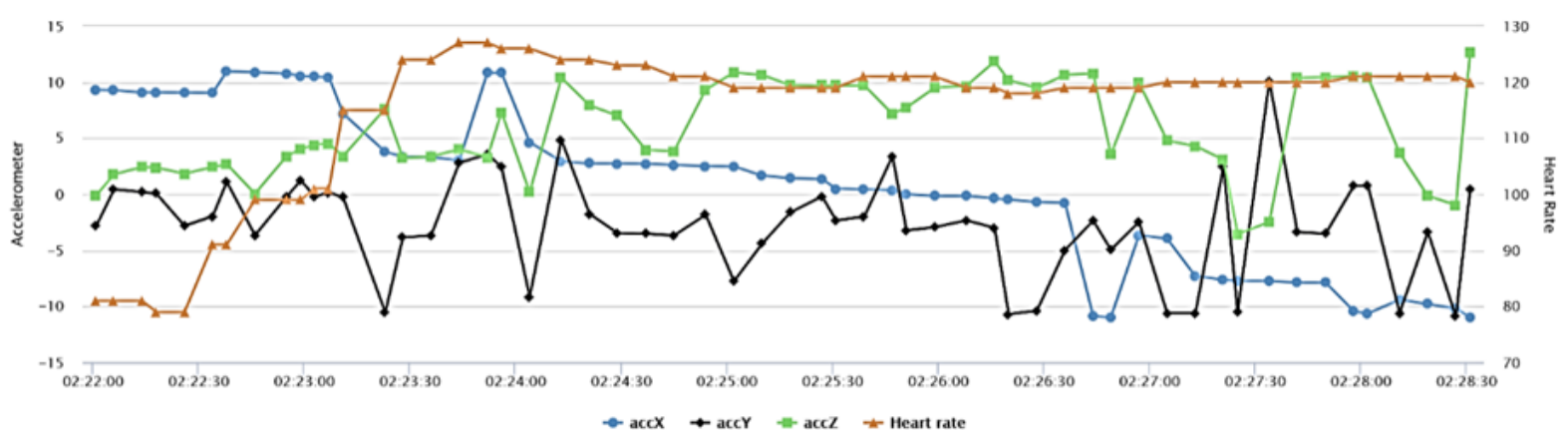

Fig. 2. Observations, Set 1 .

Both accelerometer and heart-rate sensors have been used to detect seizures in numerous previous studies [25] but in this study it was found that when used together in one device they did not always work in sync "together". This is because when the sensors were worn on the non-dominant side and a seizure occurs only the heart-rate change was indicated: the accelerometer showed no change. Yet when in correct position on the body they work in union as an excellent detection method. Therefore, demonstrating that body placement or position is paramount. For example, one patient's dominant side was the right arm. This means seizures are known to occur on the right. The results from "Observation c HP4" (Fig.2), can be seen. During shaking from the right wrist at the recorded time: 00.23am during a GTCS seizure all 3 measurements on 
axis $\mathrm{X}, \mathrm{Y}$ and $\mathrm{Z}$ showed sudden movements and the heart-rate increased to its highest peak at 128 . Before the seizure the heart-rate was much lower at 80 , then rising rapidly to 90 and up to 128. This suggests both the sensors detected the seizure correctly.

Yet the results from "Observation a HP4", seen below in Fig. 3, Observation Set 2 indicate that during a GTCS at the recorded time ' $12.44 \mathrm{pm}$ ' the 3 measurements on axis $\mathrm{X}, \mathrm{Y}$ and $\mathrm{Z}$ did not show any sudden movement, in fact barely any movement at all, yet the heart-rate increased to its highest peak at 124, in keeping with typical heart-rate increase measurement during a GTCS for HP4. Since the accelerometer was positioned on the left wrist this reveals the sensor did not detect movement therefore demonstrating the sensor was positioned in the wrong position.

Knowing the individual characteristics of the patient profile prior to sensor-based device recommendation is key, for example the HP1 with FAS (Focal Aware Seizures) and FSIA.
(Focal Seizures with Impaired Awareness): the question here was "did the 2 sensors work in union to detect the Focal seizures?" Some heart-rate increase was detected but the accelerometer was primarily redundant, for example in 'Observation 10' (Fig.3.) a seizure occurs with sensor positioned on right wrist at the observed time: $09.44 \mathrm{am}$, in this observation the heart-rate sensor detects some change over a 2 minute period i.e. The heart-rate begins at 87 increases to 90 then back to 87 then declines to 86 then steadily back to 90 . At 09.45am the heart-rate does show increase to 95 and goes back down to 85 . Heart-rate range is $87-95$, with some sudden movement from accelerometer at time of increased heart-rate.

Yet, during 'Observation 11', seen in Fig. 3 for HP1 with the seizure observed at $20.36 \mathrm{pm}$ the heart-rate range is $81-84$ with little sudden movement. Likewise, in 'Observation 12' (Fig. 4, Observations set 3) seen above: the seizure occurrence at $21.06 \mathrm{pm}$ demonstrates the heart-rate range: $81-86$ and little sudden movement.

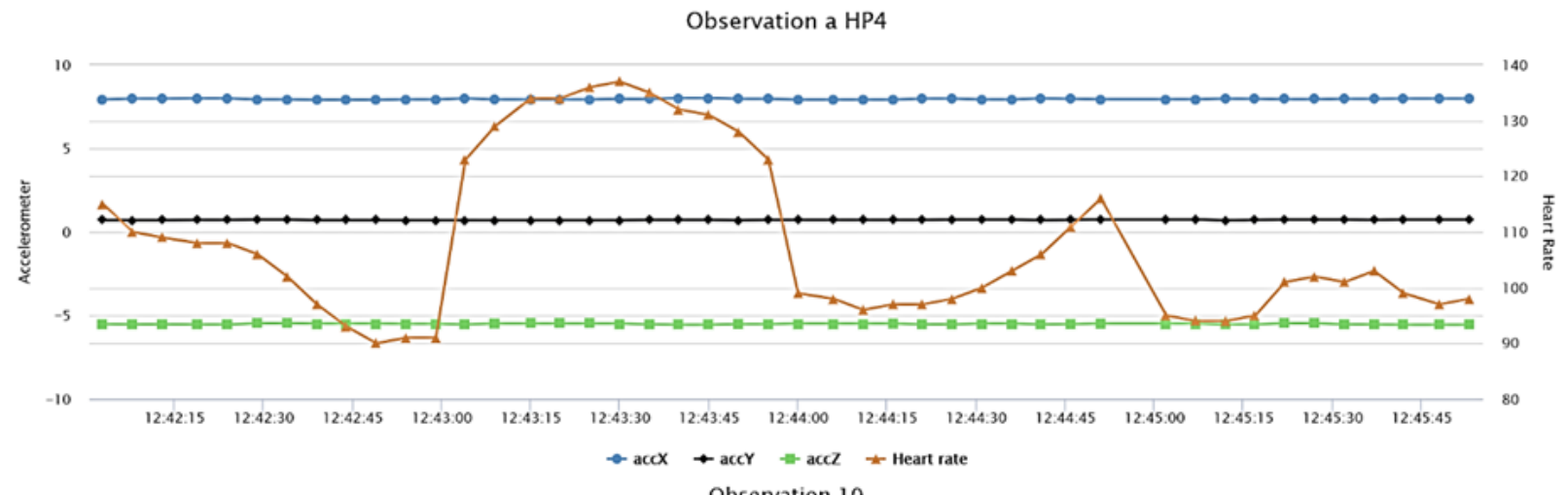

Observation 10

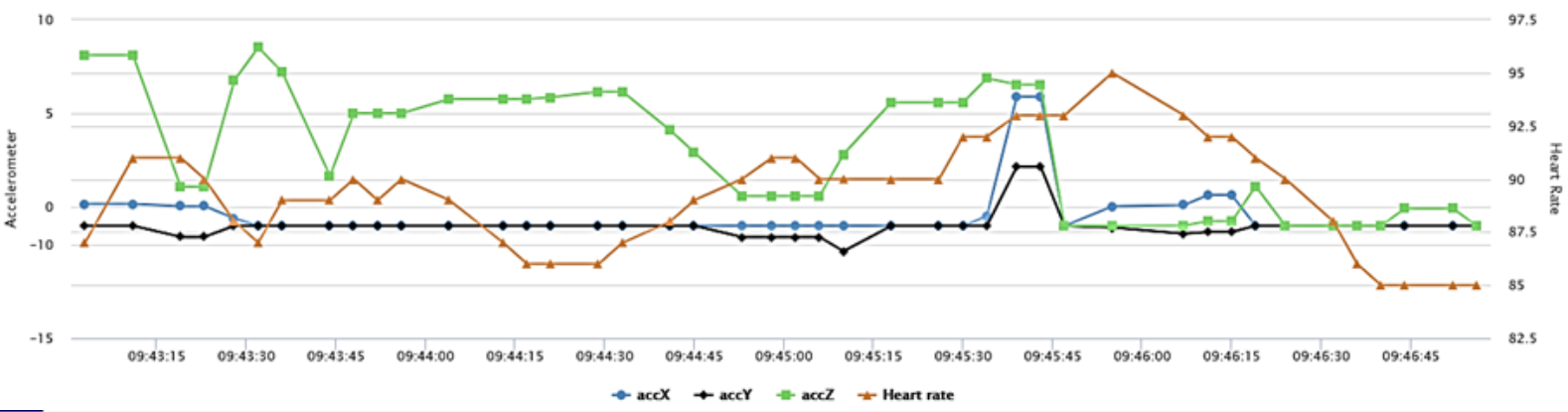

Observation 11

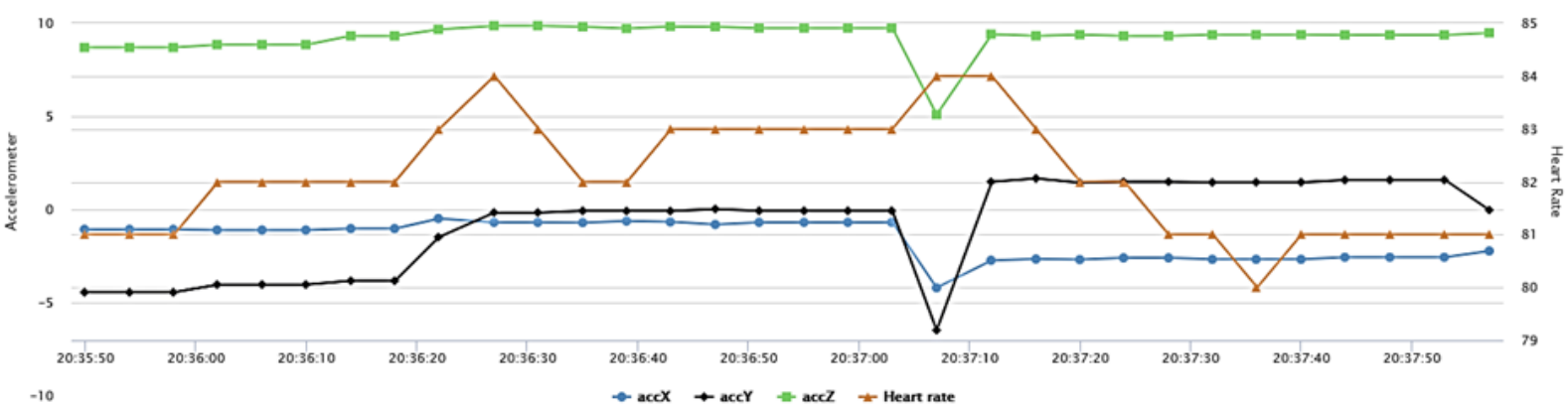

Fig. 3. Observations, Set 2. 
A pattern emerges for HP1 in other observations whereby the heart-rate decreases, for example in 'Observation a HP1' (in Fig. 4.) above at the time of the seizure '09.55am' the heartrate decreases from 90 to 84 , and likewise in 'Observation $\mathrm{f}$ HP1' (Fig.4) with heart-rate decrease from 104 to 79 during the observed time of seizure at 11.01am and in 'Observation $\mathrm{g}$ HP1' (Fig.4) with heart-rate decrease from 100 to 84 during the observed time of seizure at $20.44 \mathrm{pm}$.
In 'Observation $\mathrm{g} \mathrm{HP} 1$ ' the accelerometer indicates movement from all $3 \mathrm{X}, \mathrm{Y}$ and $\mathrm{Z}$ axis on the accelerometer at the time of the seizure. This is further observed in 'Observation e HP1' (Fig. 5, Observations, set 4) whereby there is sudden change in the accelerometer, but this is ' $21.01 \mathrm{pm}$ ' 'after' the time of seizure at ' 21.00 ' where in fact again the heart-rate shows decrease.

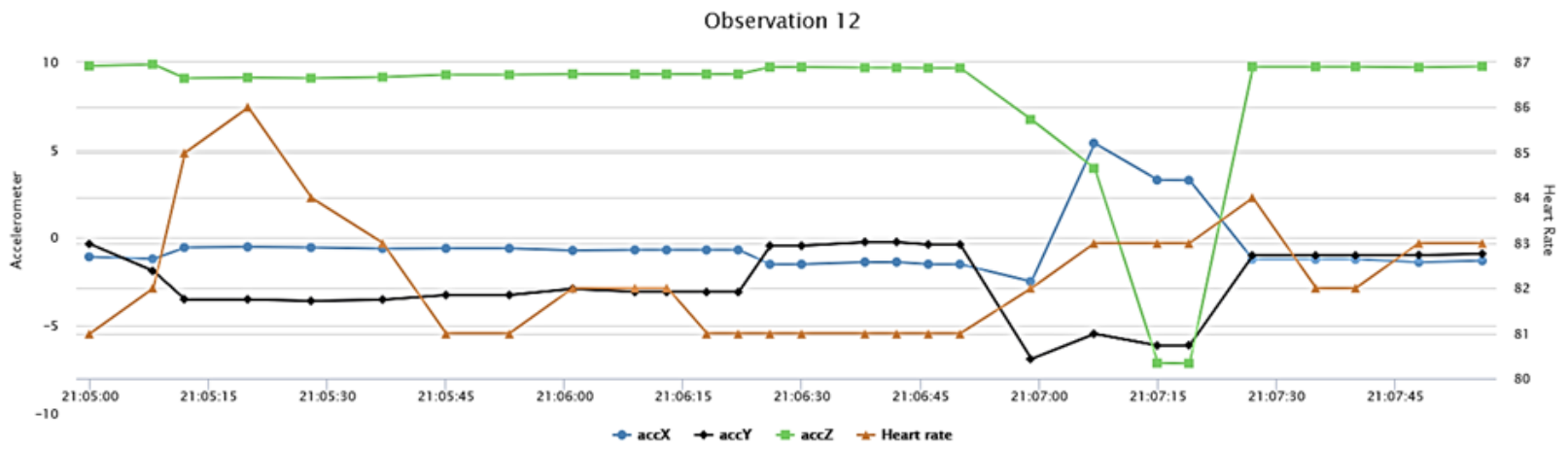

Observation a $\mathrm{HPI}$
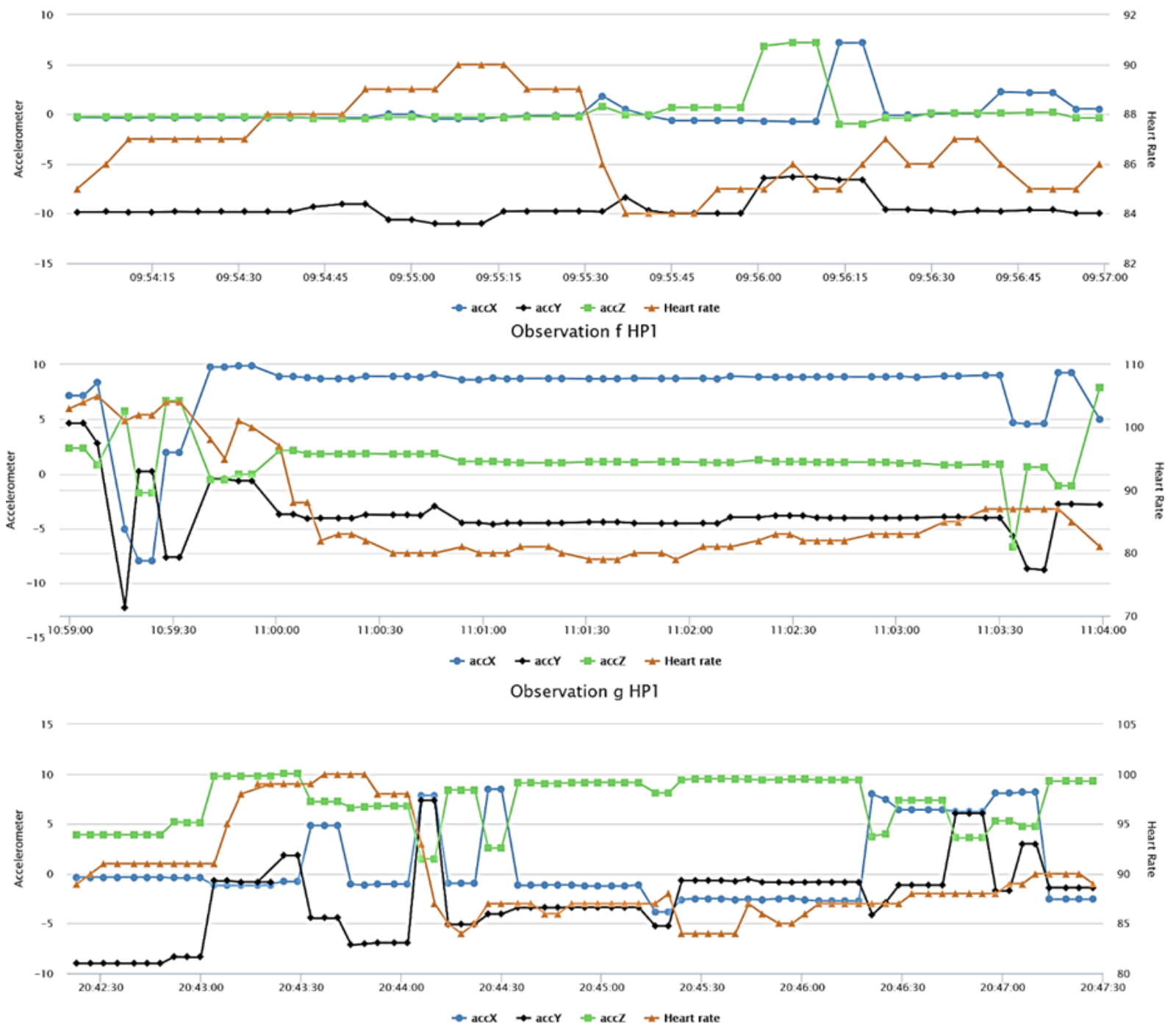

Fig. 4. Observations, Set 3. 

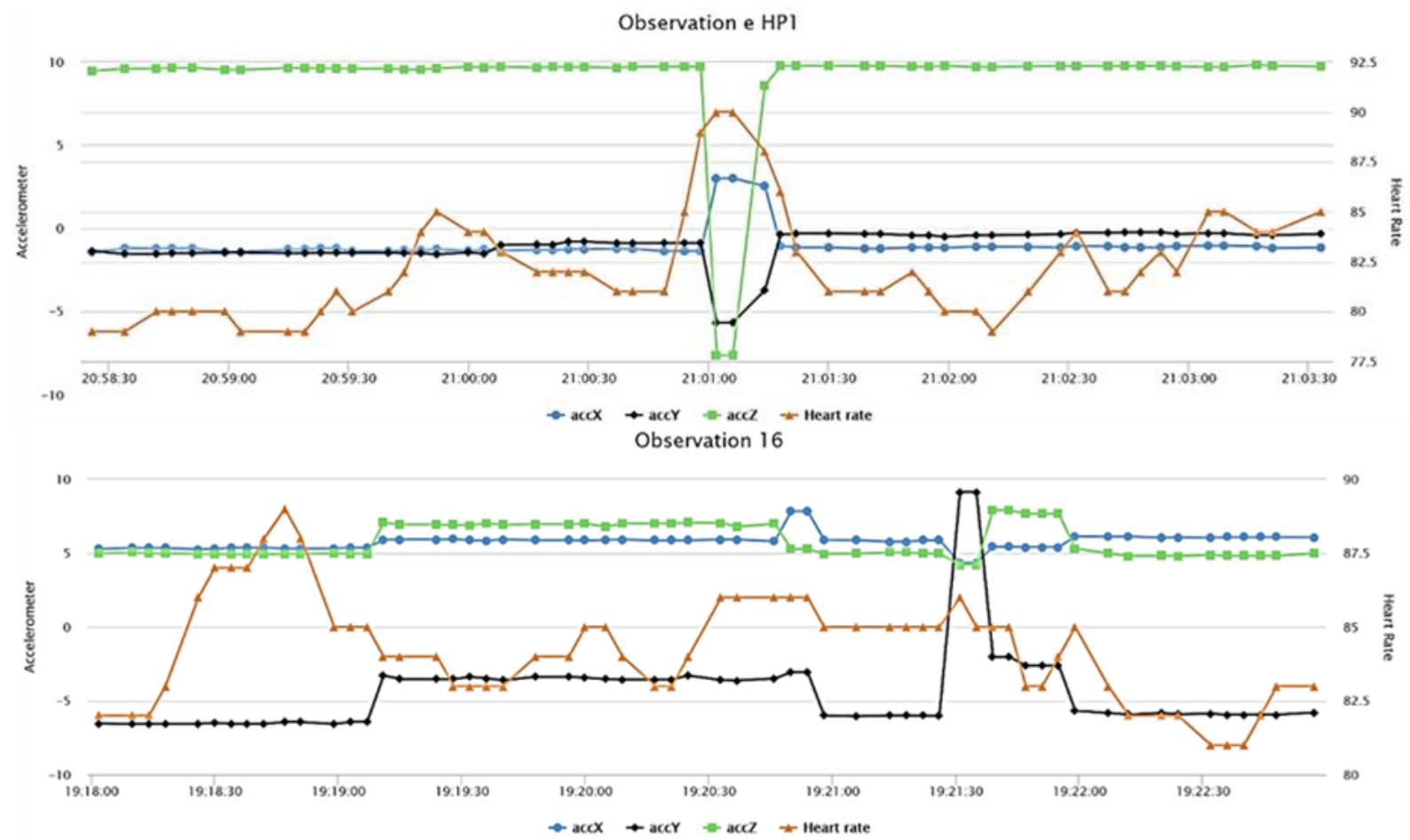

Observation 12

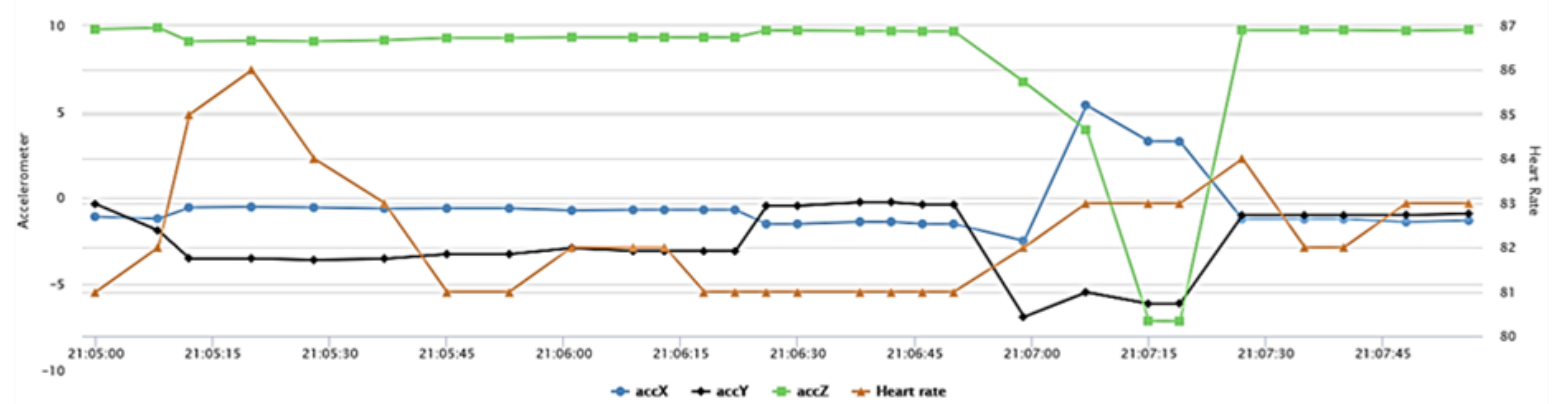

HP5. Observation 1

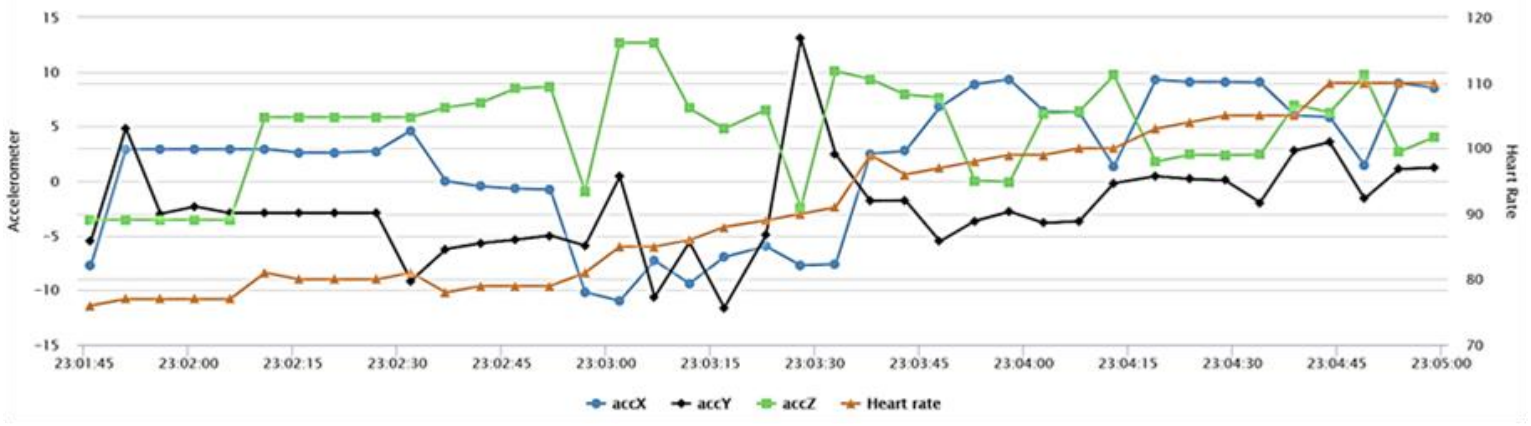

Fig. 5. Observations, Set 4.

Only 1/14 seizures observed for HP1 demonstrate significant movement for the accelerometer during seizures. Therefore, the accelerometer sensor is not useful for detecting these non-shaking seizures, although some patients' profiles (found earlier in the collection of the 'anonymous patient profiles') reveal absence seizures can evolve to convulsive generalized tonic clonic movements. Therefore, it is endorsed that Focal seizures alone, go undetected without HR changes [31].
Predominantly during non-seizure time periods for HP1 there is no significant variance in accelerometer measurements when compared to 'seizure occurrence' time periods. For example, "Observation 16" (Fig. 5) shows a 4-minute snapshot when HP1 has no seizure: the 3 axis X, Y and $\mathrm{Z}$ move similarly in "Observation 12" (Fig. 5).

Ultimately there is some evidence demonstrated here that heart-rate 'change' occurs during the seizures for HP1 
(increase and decrease). Yet since the seizures for HP1 occur for such a short time ( 20 seconds) heart-rate fluctuations can be missed or miss-interpreted as 'false alarms' perhaps due to agitation before or after the seizure.

The key and common signs and symptoms are 'LOC' (Loss of Consciousness) and 'Automatisms' for a patient such as HP1. Automatisms reveal themselves in a multitude of forms including repetitive movements, such as, lip smacking, chewing or swallowing, picking at clothes or skin or even staring [32] and these are difficult to detect with any sensor.

Hence, these repetitive movements deemed as other behavioural components of seizures which include non-motor components and post-ictal phenomena cannot be detected by the accelerometer [33] in line with the theory that seizures that are typical to the dominant body area not wearing the sensorbased device will not be detected.

Some patients exhibit automatisms such as sudden sweating events [34] and since sweating is associated with Focal Seizures a more appropriate sensor for a patient with FAS and FSIA seizure types would be Galvanic Skin Response Sensor (GSR), which refers to changes in sweat gland activity [35] as evidenced in other studies performed to detect seizures this galvanic skin response (GSR) sensor has been used in multi-modal platforms [34].

Empaticas' 'Embrace Plus' smart watch [35] can be useful for Focal seizures as it has other sensors in addition to Galvanic Skin Response (GSR), for example EDA sensor and peripheral temperature, are just one of many sensors available in this device for researchers [36]. The Electro Dermal Activity (EDA) represents the electrical changes on the surface of the skin (not just for sweat). Although witnessed in some studies finding that EDA increases during GTCS were greater than during CPS (Complex Partial Seizure, now: focal seizure with loss of consciousness) nonetheless it is a useful sensor for this type of seizure [36].

The peripheral temperature sensor also has evidence for use in detection in non-convulsive seizure's (CPS) (focal) [36].

Although the Fitbit Ionic used in this study is not a conventional device for monitoring epilepsy, it can be adapted to detect seizures as demonstrated. It is a less expensive everyday 'patient friendly' option as opposed to EEG monitoring whereby the patient wears electrodes that are not comfortable: this is because the EEG-electrodes must be attached to the scalp which hampers the patient's movement making long-term home monitoring not feasible.

In this sense this less expensive, comfortable alternative to EEG monitoring can be especially useful for patients with nonepileptic events. During early analysis in this experiment many patient profiles (found in the collected 'anonymous patient profiles') where identified as having non-epileptic events and were categorised under "Non-Classified". Although no confirmed 'epilepsy' these patients are still suffering with seizure signs and symptoms: as observed below in 'HP5. Observation 1' (Fig. 5). This patient has a non-epileptic shaking event at 23:03 pm, the observation indicates heart-rate increase from 80 up to 109 , the accelerometer also indicates rapid activity at the time of the shaking event.
One of the challenges in using sensor-based IoT devices to achieve a personalised approach is the barriers found in the use of them in hospital settings. Although EEG monitoring is the chief gold standard method used within hospitals to detect and monitor seizures, there is limited evidence found how sensorbased IoT devices and experiments are used in hospital settings.

There are very few experiments with sensor-based IoT devices that have been endorsed by the hospitals and a large problem is poor information when caring for people with epilepsy or doing epilepsy clinical trials [37] yet there is great potential to vastly increase the efficacy of epilepsy management using biomedical devices that can improve the quality of information. As available devices and sensors grow, if clinicians could be provided with more guidance in understanding and choosing which sensor suits which situation then a personalised approach can be achieved.

\section{Calibration}

Prior to this experiment upon hospital patients, this study was conducted with 2 non-epileptic volunteers who were asked to undergo the testing and perform 'simulated seizures' in a controlled environment with the sensor-based device positioned on different parts of the body at different times of the day. This was to calibrate the main hospital patient experiments. The findings are discussed below, with evidence of some of the Volunteer Observations together with the simulated individual profile characteristics and criteria used for observations.

As can be seen from 'V1.Observation 1' in Fig.5. Volunteer Observations, set 1, below, the first volunteer, with seizures occurring on the dominant right side of the body simulated a GTCS shaking from the right arm at the recorded time: $20.38 \mathrm{pm}$. The sensor-based device was worn on the right wrist. The 3 measurements on axis $\mathrm{X}, \mathrm{Y}$ and $\mathrm{Z}$ showed sudden movement and the heart-rate increased to its highest peak at 100. Before the seizure the heart-rate was steadier at 78-81, then after the seizure the heart-rate decreased to 80 . This suggested the sensors detected the simulated seizure correctly.

When V1 simulated a seizure again from the right arm they placed the sensor-based device on the left wrist at the event time: 10.10am. As identified in 'V1. Observation 3' (Fig. 6) the heart-rate shows an increase (78 to 123) yet the accelerometer axis is smooth. Similarly, in 'V1. Observation 4' (Fig.6.) the volunteer placed the device on the 'non-dominant' left leg and again the heart-rate increased dramatically but the acceleration generally smooth, although some movement on all 3 axis at the time: 09.47am of the event. Since 'some movement' was detected here a further test was performed with the device on the dominant right leg and here the difference is apparent, seen in V1. Observation 5 in Fig.7. This confirms the theory identified in earlier hospital patient observations that the sensor-based device position is paramount.

For V2 the dominant side is left. It is evident when V2 placed the sensor-based device on the left leg during a simulated seizure at $22.14 \mathrm{pm}$ both the heart-rate and accelerometer sensors are reacting vigorously, seen in 'V2.Observation 1' in Fig. 7. 
Similar to V1 when V2 places the sensor-based device on the 'non-dominant' side (right leg) during a simulated seizure only the heart-rate sensor reacts, seen below in 'V2.Observation 1a' (Fig. 7) again fueling the theory that 'position' of the sensor-based device is paramount.

\section{E. Known Characteristics}

Detection of seizures using an everyday sensor-based device and data transfer to online database was successful. This presented evidence that remote monitoring of specific epilepsy patients' profiles with known characteristics can be improved. The comfortable sensor-based device with heart-rate and accelerometer provided accurate data and is a more dependable method than a patient's paper diary.

Difference was observed due to 'position' on the body of the sensor-based device, demonstrating that because of the known patient specific characteristics a personalised approach is achieved. Furthermore, it was discovered that the 'type' of sensor used is principal in its correspondence with a patients' particular 'seizure type' together with the particular associated signs or symptoms.

The sensors and techniques used in this experiment enable some assurance in long term remote monitoring. The use of such sensor-based device used in this experiment can reduce the frequency of visits to hospitals and improve daily management of epilepsy thus, these sensing techniques have shown that results can be achieved in the measurement of specific epileptic seizures based on observations.

As established through these experiments' timely detection along with known patient characteristics is one of the keys to monitoring epilepsy.

\section{V1. Observation 1}

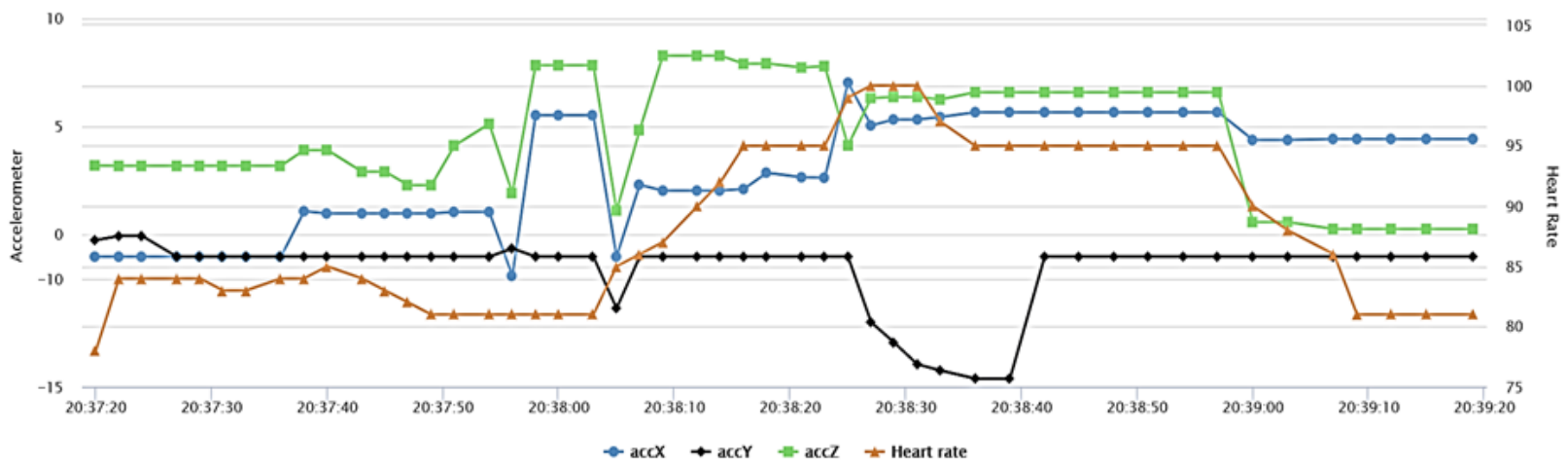

V1. Observation 3

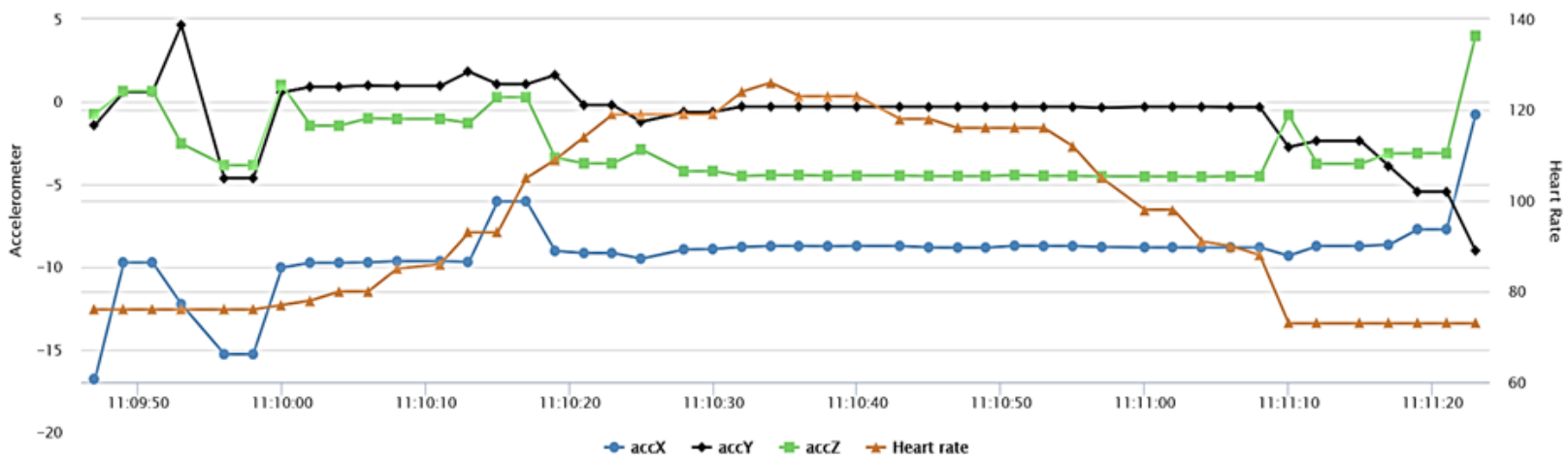

V1. Observation 4

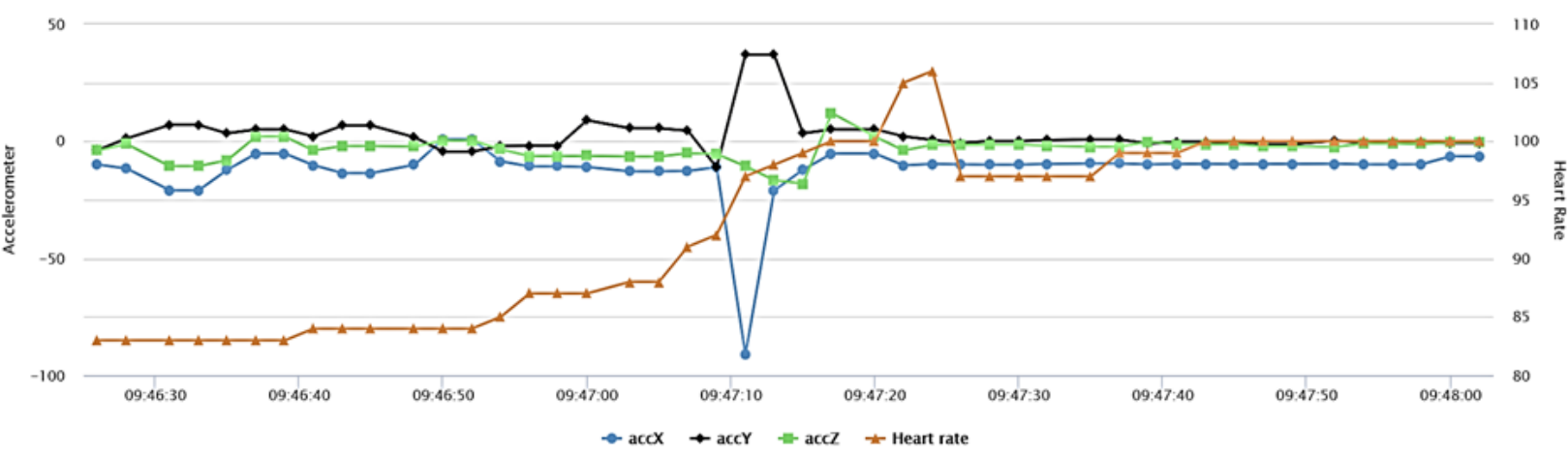

Fig. 6. Volunteer Observations, Set 1. 
VI. Observation 5
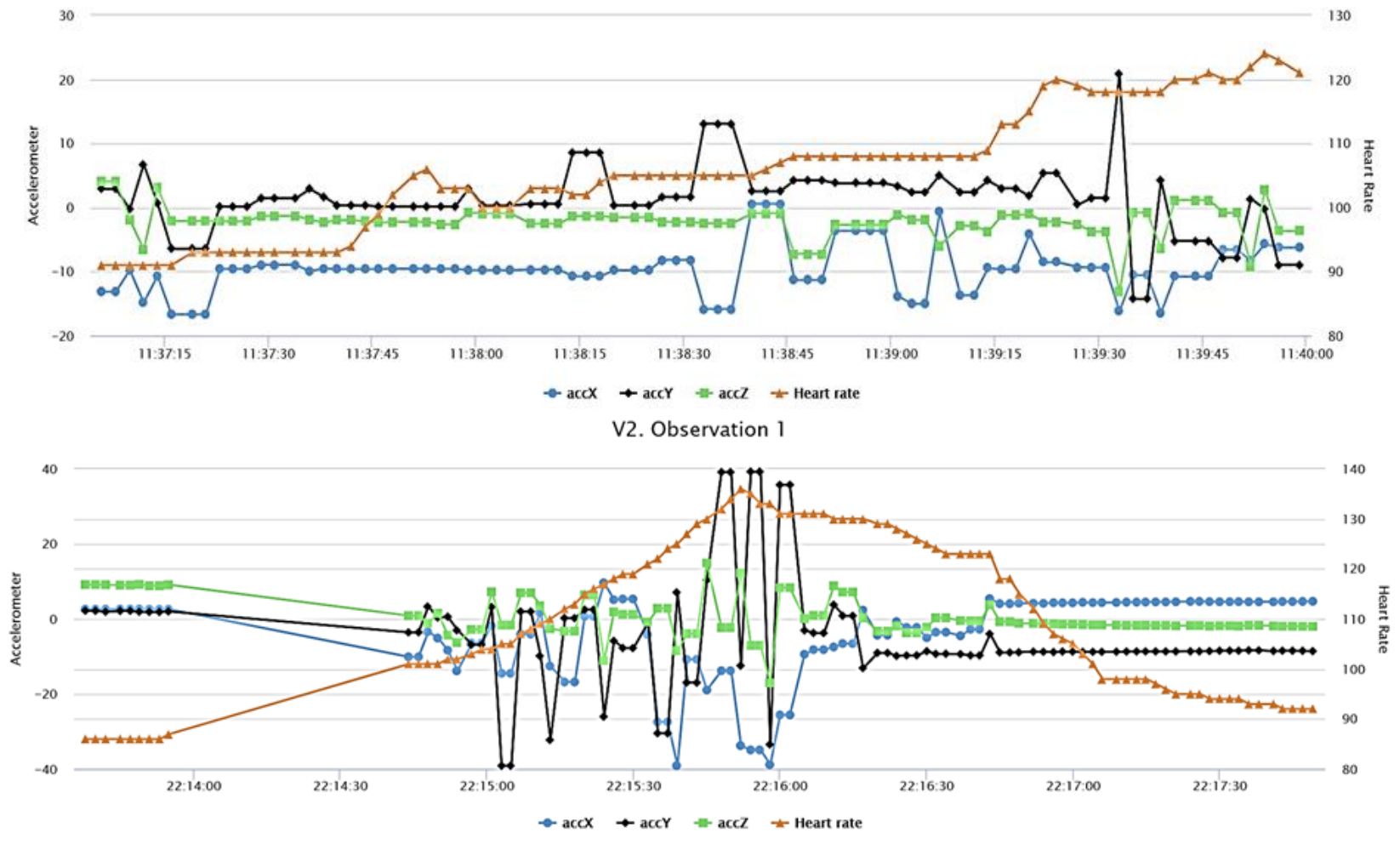

V2. Observation 1a

15

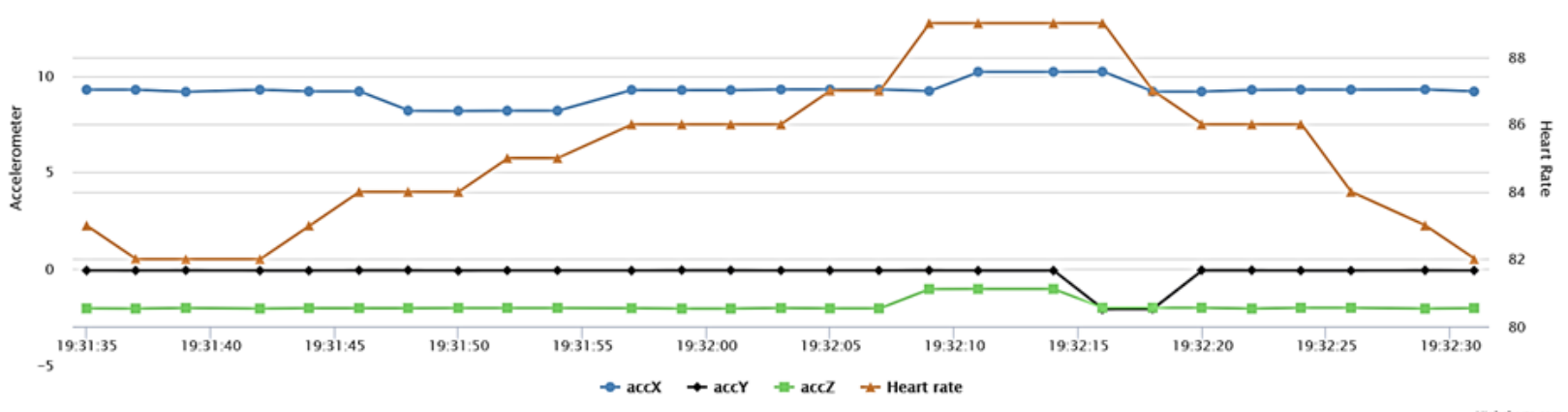

V1. Observation 4

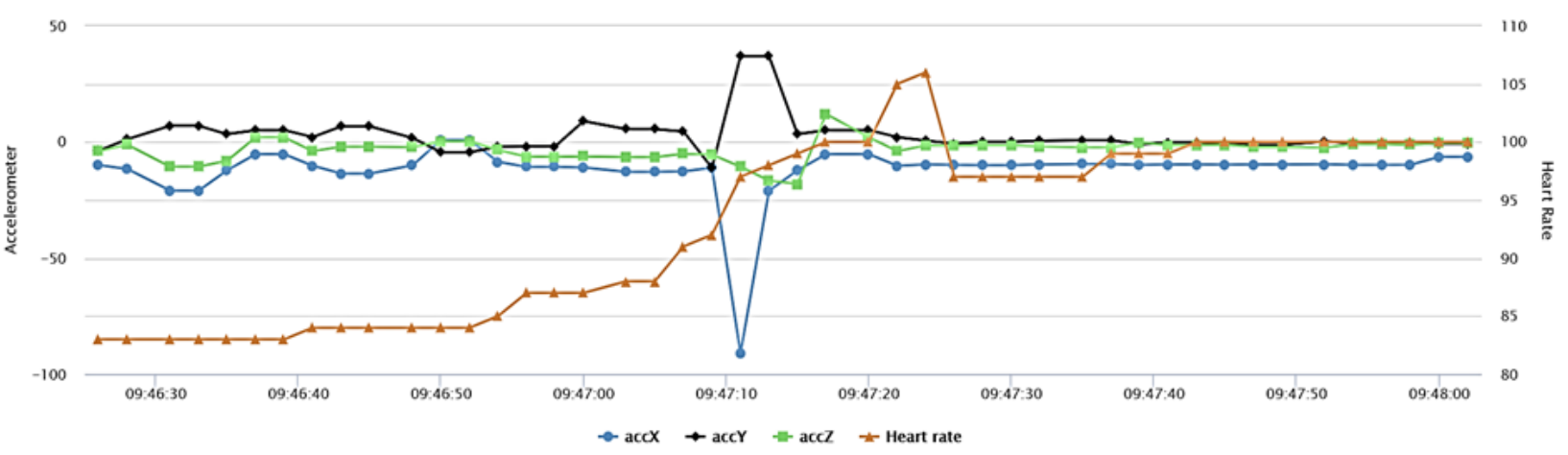

Fig. 7. Volunteer Observations, Set 2. 


\section{IOT BASED EPILEPSY MONITORING MODEL}

The purpose of the IoT based Epilepsy monitoring model [38] is to support a 'Personalised Monitoring Plan' framework in collecting data from a variety of potential epilepsy device sensors and also provide optimal analysis tools to utilise the sensor data thus supporting clinicians to monitor epilepsy patients.

\section{A. PMP Framework}

This section proposes a Personalised Monitoring Plan (PMP) framework. In the previous section experiments were performed to capture seizure data, obtained from sensors, which are positioned on different parts of the patient's body. The results from this experiment are used to inform a PMP (Personalised Monitoring Plan), seen below in Fig. 8 which recommends which sensor-based device to use based on those very individual, personal characteristics of a given patient.

The proposed 'Personalised Monitoring Plan' (PMP) framework is a model for which doctors and healthcare professionals (HCPs) can use to assist in identifying which device they should recommend to the individual patient for remote monitoring.

The PMP framework integrates two types of 'personalisation:

- The patient as the individual (derived from an ontology language).

- Use Patients in a category (using the K-means Clustering method).

Both these personalisation elements are described below in in the next sections. The third tool of the PMP framework supports the decisions surrounding recommending the correct IoT sensor-based devices. The main purpose is to help HCPs decide which IoT Sensors to recommend for monitoring and which position on the patient's body.

The PMP framework ultimately allows users to provide a description of the 'seizure condition' of a single patient or a patient type, and to automatically obtain a PMP adjusted to the patient requirements.

The proposed framework consists of two features: the first being 'Personalisation' (based on this study) and the second is the anticipated 'Remote Monitoring', shown in pink and blue respectively in Fig. 8, PMP Framework.

\section{B. Personalisation Elements}

The personalisation contributions are part of the preceding findings in this study. The ontology language was created to support the need of the healthcare process to transmit, re-use and share individual patient profile data related to their seizures.

The ontology was achieved by the initial examination of 100 anonymous epilepsy patient medical records. The data was analysed to discover if values for each of the attributes are different for each patient, together with the investigation of epilepsy 'terminology' and existing seizure type classifications/categories were analysed so that an 'individual' seizure type patient profile could be formed. A close collaboration with clinicians helped to build a data model fit for real-world adoption inside hospital settings and thus an ontology was developed to model the concept of the epilepsy patient profile, namely ESO 'Epilepsy Seizure Ontology'. This was a driving force for the PMP Framework and a critical aspect for this concept. In order to make ESO useable for HCPs (Health Care Professionals) the ontology was transformed into a language that is understandable by humans and machines, this was accomplished by XML and the outcome was PPDL (Patient Profile Description Language).

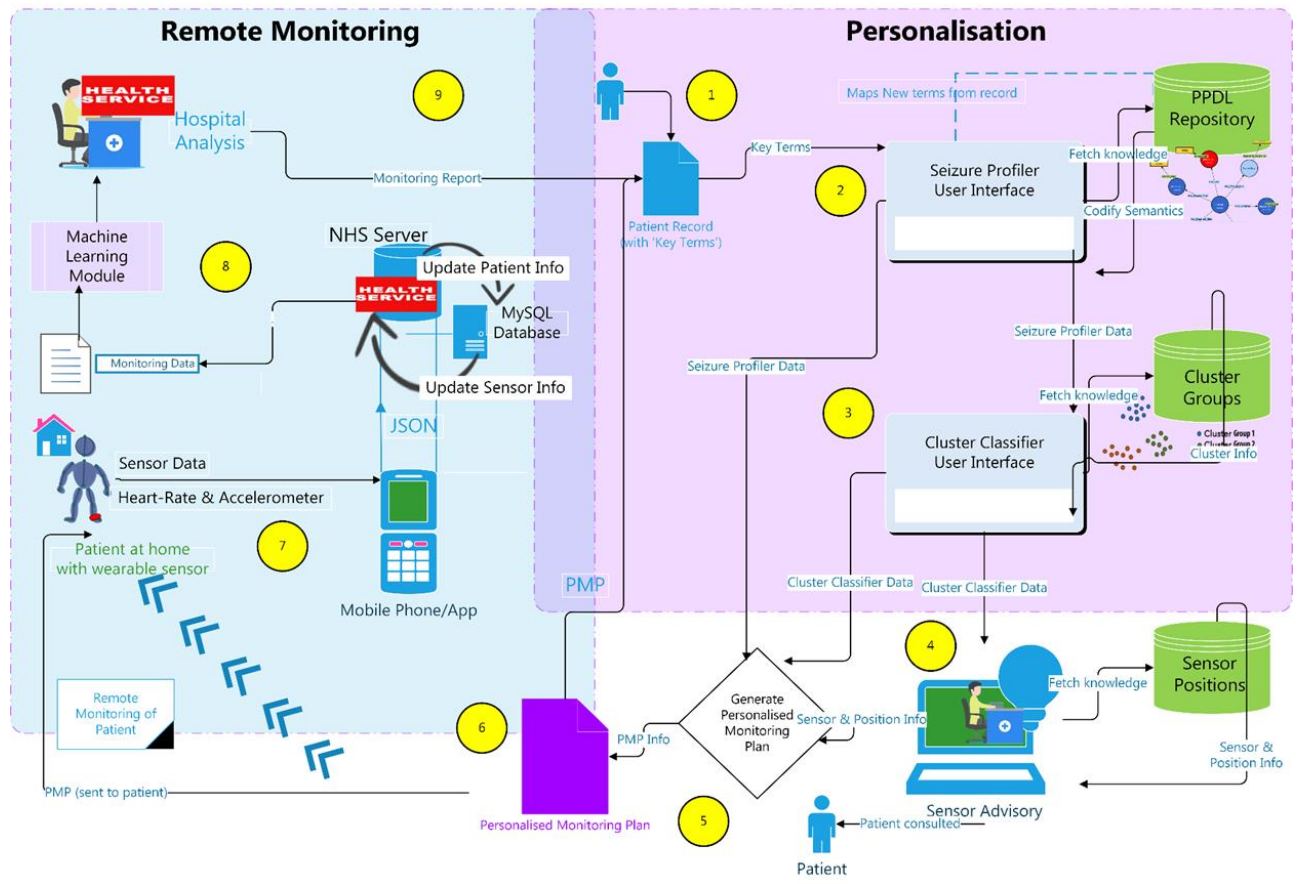

Fig. 8. PMP Framework. 
The second personalisation element was achieved by using K-means Clustering analysis. Different clustering techniques were initially analysed to find the most appropriate approach for the acquired epilepsy data and an in-depth focus upon 'clustering considerations' was undertaken to confirm validity.

The outcome was a set of 6 distinct 'clustering' groups, shown in Table I. These 6 cluster groups revealed six completely different categories of patients each with their distinct seizure related information.

The results revealed the distinct groups of epilepsy patients that share similar characteristics using Clustering Analysis. This will enable the health carers to define a 'type' of epilepsy patient.

\section{PMP Framework Loop and Maintenance}

With this PMP framework, the PPDL (Patient Profile Description Language) can be directly maintained (and extended) by HCP's. The framework has the flexibility, (as the ontology grows with new seizure related concepts), to deal with the mounting diversity of seizure type patients. Therefore, the PMP framework is somewhat reliant on the integration of new knowledge in the PPDL. As the HCP of the PMP Framework approves the recommendations, the information about the patient and the advice may change and this new information can cause the PMP Framework to continue providing new suggestions to the HCP.

This loop will stop either when the framework is not able to provide new recommendations or when the HCP considers that the current condition of the patient is correctly represented by the recommendation. At any time, the PMP for the patient is fundamentally controlled by the $\mathrm{HCP}$ who is using the framework.

Consequently both the personalised 'seizure related data' and the 'cluster classifier data' of a patient may evolve as the patient disorder changes, for example when the information about the patient changes in the patient record of that patient or as a result of the application of the PMP Framework to find out new 'seizure type' knowledge about the current patient. The datasets are expected to evolve and are continuously stored as part of the record of that patient.

\section{IoT based Epilepsy Monitoring Model}

To achieve the type of monitoring described in the PMP framework, several IoT components can be deployed to retrieve sensor data from the epilepsy patient to be accessed remotely. These components include the integration of the personalisation components described in the PMP framework, those of an internet connection and protocols which form the 'network layer', a cloud platform to manage the data analysis and fundamentally the sensor-based devices forming the sensor layer. These components make the ingredients of an IoT solution, proposed in the IoT based Epilepsy monitoring model shown in Fig. 9.

The sensor layer, (discussed in section F) has the task of acquiring and sending the data from the different epilepsy devices involved in capturing seizure data, to the proposed cloud platform.

The 'IoT based Epilepsy monitoring model' proposal in Fig.9 shows areas on the body where parameters are measured, each area is indicated with a colour matching the parameter.

\section{E. Cloud Platform}

The proposed cloud platform provides all the necessary services for the clinician to manage, process and visualise the seizure data. All the processes that involve the interaction between the personalisation layer and the sensor layer are carried out through the following modules: PMP data management, machine learning module and data analysis \& visualisation. All these services are hosted in the cloud and clinicians are able to access them remotely from any location.

The data analysis and visualisation module utilises the sensor data while the 'PMP data management' module pulls all the patient records from the personalisation modules and here the sensor data results are updated. Visualisation is a requirement for any such system as it is important for clinicians to be provided with user friendly GUIs so they can study the seizure data from the epilepsy sensor devices. The machine learning module is also proposed, this is a key aspect for future development and the idea is that by using algorithms the module will 'learn' when a patient is about to have seizure and warn them in advance.

TABLE I. CLUSTER GROUPS

\begin{tabular}{|l|l|l|l|l|l|l|}
\hline Attribute & Cluster & & & & \\
\hline & Cluster 0 & Cluster 1 & Cluster 2 & Cluster 3 & Cluster 4 \\
\hline Seizure Type & NMA & Un-classified & FAS & Gelastic & GTCS \\
\hline $\begin{array}{l}\text { Key Sign / } \\
\text { Symptoms }\end{array}$ & LOC & LOC & Sensations & None & $\begin{array}{l}\text { Myoclonus } \\
\text { Bilateral } \\
\text { Clonus }\end{array}$ \\
\hline & $\begin{array}{l}\text { Urinary / In- } \\
\text { continence }\end{array}$ & None & & $\begin{array}{l}\text { Bilateral } \\
\text { Clonus }\end{array}$ & LOC \\
\hline $\begin{array}{l}\text { Common Sign / } \\
\text { Symptoms }\end{array}$ & Automatism & $\begin{array}{l}\text { Cognitive } \\
\text { Automatism }\end{array}$ & Automatism & Automatism & Sensory Cognitive & Automatism \\
\hline Arm / Leg & Either & Leg & Either & Either & Leg & $\begin{array}{l}\text { Nocturnal and } \\
\text { Diurnal }\end{array}$ \\
\hline Nocturnal / Diurnal & $\begin{array}{l}\text { Nocturnal and } \\
\text { Diurnal }\end{array}$ & $\begin{array}{l}\text { Nocturnal and } \\
\text { Diurnal }\end{array}$ & Diurnal & Diurnal & \\
\hline
\end{tabular}




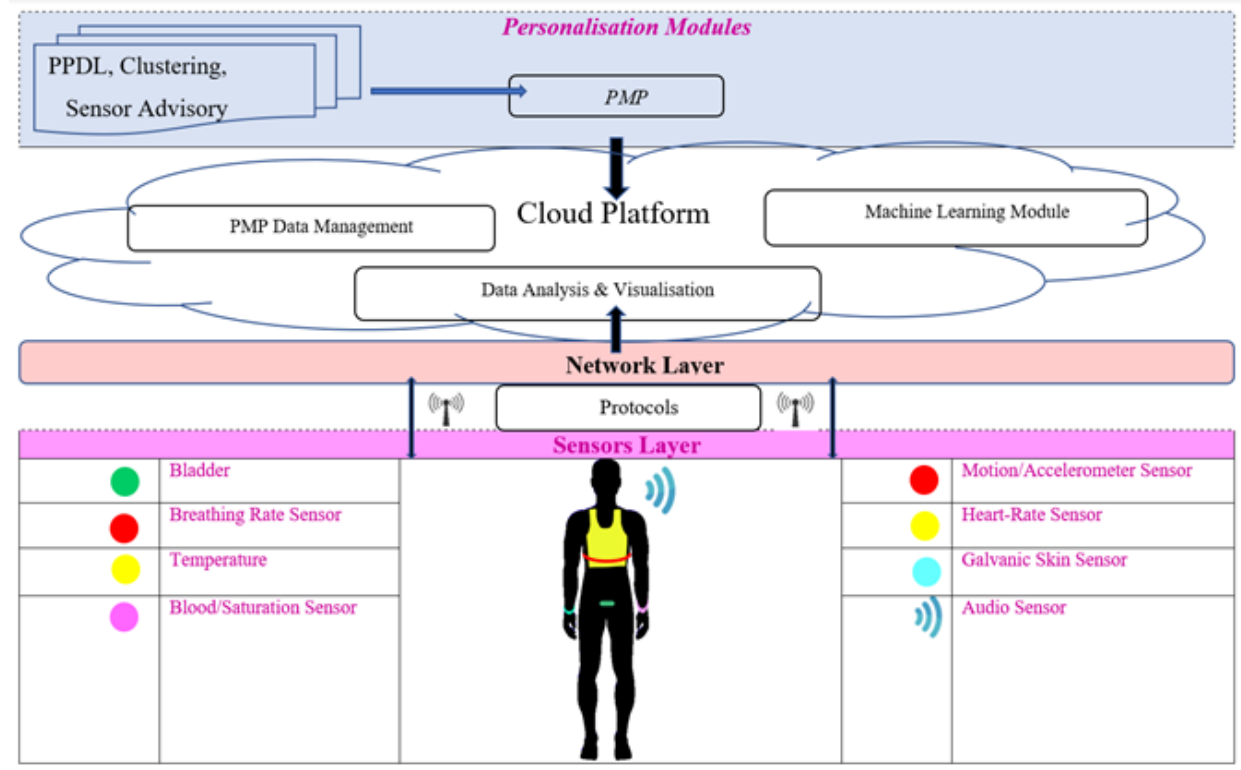

Fig. 9. IoT based Epilepsy Monitoring Model.

A pre-processing hardware and a platform are needed to communicate and transmit the sensor data which is collected using wearable sensors positioned on a patient's body. The Microsoft Azure IoT platform [39] is proposed, since this cloud computing server is trusted and safe [40].

\section{F. Sensor Layer}

The sensor layer has the task of acquiring and sending the data from the different epilepsy devices involved in capturing seizure data, to the proposed cloud platform.

The sensors previously used in the experiment, the heartrate and accelerometer sensors were demonstrated within the PMP framework discussed in this paper. Yet there are other sensors too that can work within the context of this research. These potential sensors found in other devices are explored and proposed below.

Despite the expense, multi-modal sensor based devices are the ultimate desire to monitor an epilepsy patients seizures since multiple sensors are embedded in one device and make comfortability for the patient and all-in-one solutions for the manager of the device, furthermore epilepsy patients have revealed their preference for devices capable of monitoring several parameters [24]. Section III in this paper uncovered some of the epilepsy detection devices and monitoring systems, whilst section IV demonstrated the use of an inexpensive device with heart-rate and accelerometer sensor (justified for experimental purposes), and simultaneously identified other useful sensors for epilepsy. These other sensors which exist in devices, those that go beyond heart and movement sensing, and beyond fitness devices, are amongst a vast amount. Consequently, many studies have analysed the performance and limitations of each sensor based device, one critical evaluation by Peake [41] found many devices where not yet fully validated or tested for reliability, therefore this examination will aim only to propose validated devices for use in the IoT based Epilepsy monitoring model.
Recently in 2020, Abreu, Fred et al [42] did a significant exploration on, wearables and related devices, that can be utilised for epilepsy prediction, the findings presents devices, some with multiple sensors, characterised with respect to their applicability to research, validation status, form factor or body positioning, battery duration, method to access the data, measured signals and, their applicability to epilepsy prediction (EP) [42]. This is a vital study since the devices have already been validated, and connectivity options identified, and since they are beneficial for epilepsy they can be proposed in the IoT based Epilepsy monitoring model.

These devices and their sensors, identified as applicable for epilepsy prediction contain some of the sensors that were highlighted during the experiments i.e. accelerometer, heartrate and GSR, but the audio and bladder sensors were not previously included. In this case the audio and bladder are added to the IoT based Epilepsy monitoring model in Fig.9 above and the connectivity options and battery life can be referred to in Table II.

The IoT based Epilepsy monitoring model proposal in Fig.9 shows areas on the body where parameters are measured, each area is indicated with a colour matching the parameter. The chosen devices have been proposed based upon the factors in the study by Abreu, Fred et al [42] for the best battery life, validity, and connectivity options selected for ease of connection to the cloud platform in the IoT based Epilepsy monitoring model. Since Embrace2 device uses its own onboard processing it is not adaptable for the model proposed in this study. Furthermore some of the device based sensors depicted in

Table II. 'Selected Sensors' are multi-modal so their use can sometimes be proposed in more than one activity area, for example the device EQ02 has both the heart-rate and temperature sensor. 
TABLE II. SELECTED SENSORS

\begin{tabular}{|l|l|l|l|l|}
\hline Body Area & Validated Device & Sensor & Connectivity Option & Battery Life \\
\hline Breathing & HexoSkin & RESP & Cloud Storage or BLE transmission & $12 \mathrm{~h}$ \\
\hline Electrodermal & Empatica E4 & EDA or GVS & Cloud Storage & $48 \mathrm{~h}$ \\
\hline Blood Volume & Empatica E4 & PPG & Cloud Storage & $48 \mathrm{~h}$ \\
\hline Movement & Bioharness3 & ACC & BLE transmission & $12-24 \mathrm{~h}$ \\
\hline Heart-Rate & EQ02 & ECG & Bluetooth transmission & $48 \mathrm{~h}$ \\
\hline Temperature & EQ02 & Temp & Bluetooth transmission & $48 \mathrm{~h}$ \\
\hline Audio & Alert-it & Sound Sensor & Ethernet connection & $12-16 \mathrm{~h}$ \\
\hline Bladder/ Incontinence & DFree & Ultra- sound & Bluetooth transmission & $24 \mathrm{~h}$ \\
\hline
\end{tabular}

\section{G. Network Layer}

There are several ways the sensors can connect and send data to the cloud platform and since most of the sensor devices connect to a mobile phone they are served by Bluetooth or Bluetooth Low Energy (BLE) and use very little power. Nevertheless, each sensor-based device is provided with its own protocol and connectivity options, hence the type of IoT connectivity is determined generally by the distance that the data must travel, either short-range or long-range [43]. IoT platforms such as Azure use gateways to connect IoT devices to the cloud. The data collected from the devices moves through this gateway, gets pre-processed using in build modules (Edge) and then gets sent to the cloud. Data is protected by an additional layer of security provided by the Azure Application gateway and in addition connection security is enabled as each connected IoT device is given a unique identity key [39].

\section{Evaluation}

An evaluation was performed by taking two different epilepsy patients through the steps in the PMP framework. Two different 'use case' scenarios each with different patient profiles were tested by revealing their respective inputs and outputs. The aim was to provide the Personalised Monitoring Plan (PMP) described in this study and only the 'seizure related' information of the patient was considered. This input data is primarily composed of the patients' seizure types, signs and symptoms. Therefore, given the condition of an epilepsy patient, the PMP is used to personalise the medical knowledge available for that patient, all other unrelated medical knowledge to the patient is discarded. The input data was processed using the framework features: in summary the framework produced new datasets that were passed to the 'Generate PMP' component, these were: seizure profiler data, cluster classifier data and sensor position information. The evaluation results helped determine the effectiveness of the PMP framework and how it can be used as a tool for recommending the IoT device to an epilepsy individual patient.

\section{VII.LONG TERM USES AND APPLICABILITY IN OTHER DOMAINS}

The methods used in this study for ontology development and clustering analysis can be applied to any disease whereby recognised symptoms per patient can be individualised and be further put into sub-groups or categories. However, to fully utilise this personalised approach the application of the PMP framework can be particularly applied to patients whom have symptoms that can be monitored with different IoT sensorbased devices and personalised further by wearing the device on different body positions. In the future the following types of patients can be handled by the proposed PMP framework: (shown below together with latest progressive recommended sensor-based devices).

- Diabetes: i.e. One such recommended device could be use of 'flash glucose sensing': A device which checks blood glucose levels by scanning a sensor worn on their arm will be (available on the NHS for people with type 1 diabetes) [44].

- Sick Infants: i.e. A recommended device could be use of a miniaturised, wireless oxygen sensor wearable device the size of a Band-Aid which would allow babies to be monitored from home and able to leave the hospital [45].

- Rehabilitation: i.e. the recommended device for rehabilitation could be a Force-based sensor which can be integrated with footwear to measure the interaction of the body with the ground during walking [36]. Due to the possibility of detecting not only physiological but also movement data wearable sensors have also acquired increasing importance in the field of rehabilitation [46].

\section{A. Machine Learning}

Another significant future direction for long-term remote monitoring of epilepsy is seizure detection via 'machine learning'. By accumulating large datasets, computers can learn by recognising patterns in data.

This automated approach (without human intervention) has been proposed as a 'machine learning module' within the PMP framework and IoT based Epilepsy monitoring model to determine seizure detection or not based on the patient specific profile, the idea being that by using algorithms the module will 'learn' when a patient is about to have seizure and warn them in advance. Due to limitations in this study this module has not been built but instead is proposed at the next stage and recommended for the next level. Largely there is further work to take the PMP conceptual model and the IoT based Epilepsy monitoring model into full operation.

Recent advances in machine learning and deep learning technique inventions have shown noteworthy advantage in the 
automatic learning of robust features that outperformed the human oriented features in many domains such as self-driving cars, natural language processing, and computer vision also medical diagnosis [47] [48].

Yet as identified in a review of epileptic seizure detection using machine learning classifiers a major challenge is to detect seizures correctly from a large volume of data [49], and it is highlighted that the selection of suitable classifiers and features are crucial [49].

Ultimately, along with the challenges associated with the increasing dataset sizes (hence growing epilepsy cases), and evolving date science hitches, as well as obtaining sensitive data it could be argued that the greatest challenge of all to help solve these problems is the enabling collaboration between people with differences in expertise [50].

\section{CONCLUSION}

The principal contribution in this study was that with the prior 'knowledge' of individual patient characteristics drawn from the PPDL repository and 'Cluster Groups' together with the supplementary 'proof of concept' knowledge obtained in the experiments each epilepsy patient can be treated distinctly and recommended an appropriate sensor-based device thus forming a patient specific unique PMP (Personalised Monitoring Plan). Hence personalisation can be achieved.

The sensors and techniques used in the experiment enables some assurance in long term remote monitoring. The use of such sensor-based device used in the experiment can reduce the frequency of visits to hospitals and improve daily management of epilepsy thus, these sensing techniques have shown that results can be achieved in the measurement of specific epileptic seizures based on observations.

As established through these experiments' timely detection along with known patient characteristics is one of the keys to monitoring epilepsy.

The integration of the components and technologies in the framework depicted in Fig.8 PMP Framework aims at providing HCP's dealing with epilepsy patients with an integrated tool that helps them in recommending the correct IoT sensor and position on the patient's body.

These decisions are made at the initial consultation and act as an 'aid' in personalising the condition of new incoming patients, and thus refine the predefined 'patient record' in order to obtain and validate a 'Personalised Monitoring Plan' which is in addition adapted to include the seizure monitoring of the patient during appointments.

The PMP Framework is designed to provide a patientempowering support in a way that the available knowledge is continuously personalised to the condition of the seizure type patient. The IoT based Epilepsy monitoring model has been proposed and can be adopted by the PMP framework in future developments.

\section{REFERENCES}

[1] M. Pediaditis, M. Tsiknakis, V. Kritsotakis, M. Goralczyk, S. Voutoufianakis, and P. Vorgia, "Exploiting advanced video analysis technologies for a smart home monitoring platform for epileptic patients: Technological and legal preconditions," presented at the 2012 International Conference on Telecommunications and Multimedia (TEMU), 2012/07, 2012. [Online]. Available: http://dx.doi.org/10.1109/temu.2012.6294719.

[2] N. Moghim and D. W. Corne, "Predicting epileptic seizures in advance," (in eng), PLoS One, vol. 9, no. 6, pp. e99334-e99334, 2014, doi: 10.1371/journal.pone.0099334.

[3] B. S. Chang and D. H. Lowenstein, "Epilepsy," New England Journal of Medicine, vol. 349, no. 13, pp. 1257-1266, 2003/09/25 2003, doi: 10.1056/nejmra022308.

[4] D. Hirtz, D. J. Thurman, K. Gwinn-Hardy, M. Mohamed, A. R. Chaudhuri, and R. Zalutsky, "How common are the "common" neurologic disorders?," Neurology, vol. 68, no. 5, pp. 326-337, 2007/01/29 2007, doi: 10.1212/01.wnl.0000252807.38124.a3.

[5] L. Chen et al., "OMDP: An ontology-based model for diagnosis and treatment of diabetes patients in remote healthcare systems," International Journal of Distributed Sensor Networks, vol. 15, no. 5, p. 155014771984711, 2019/05 2019, doi: 10.1177/1550147719847112.

[6] A. F. Van Straten and B. C. Jobst, "Future of epilepsy treatment: integration of devices," Future Neurology, vol. 9, no. 6, pp. 587-596, 2014/11 2014, doi: 10.2217/fnl.14.54.

[7] M. Tentori, L. Escobedo, and G. Balderas, "A Smart Environment for Children with Autism," IEEE Pervasive Computing, vol. 14, no. 2, pp. 42-50, 2015/04 2015, doi: 10.1109/mprv.2015.22.

[8] T. Tamura and W. Chen, Seamless healthcare monitoring. Springer, 2018.

[9] P. Bonato, "Wearable Sensors and Systems," IEEE Engineering in Medicine and Biology Magazine, vol. 29, no. 3, pp. 25-36, 2010/05 2010, doi: $10.1109 / \mathrm{memb} .2010 .936554$.

[10] E. Magiorkinis, A. Diamantis, K. Sidiropoulou, and C. Panteliadis, "Highights in the history of epilepsy: the last 200 years," (in eng), Epilepsy Res Treat, vol. 2014, pp. 582039-582039, 2014, doi: $10.1155 / 2014 / 582039$.

[11] D. J. Cook, M. Schmitter-Edgecombe, and P. Dawadi, "Analyzing Activity Behavior and Movement in a Naturalistic Environment Using Smart Home Techniques," (in eng), IEEE J Biomed Health Inform, vol. 19, no. 6, pp. 1882-1892, 2015, doi: 10.1109/JBHI.2015.2461659.

[12] T. D. Parsons and R. L. Kane, "Computational Neuropsychology," in The Role of Technology in Clinical Neuropsychology, ed: Oxford University Press, 2017.

[13] V. J. Aski, S. S. Sonawane, and U. Soni, "IoT Enabled Ubiquitous Healthcare Data Acquisition and Monitoring System for Personal and Medical Usage Powered by Cloud Application: An Architectural Overview," in Advances in Intelligent Systems and Computing, ed: Springer Singapore, 2018, pp. 1-15.

[14] B. Direito, C. Teixeira, B. Ribeiro, M. Castelo-Branco, F. Sales, and A. Dourado, "Modeling epileptic brain states using EEG spectral analysis and topographic mapping," Journal of Neuroscience Methods, vol. 210, no. 2, pp. 220-229, 2012/09 2012, doi: 10.1016/j.jneumeth.2012.07.006.

[15] S. Xie and S. Krishnan, "Wavelet-based sparse functional linear model with applications to EEGs seizure detection and epilepsy diagnosis," Medical \& Biological Engineering \& Computing, vol. 51, no. 1-2, pp. 4960, 2012/10/09 2012, doi: 10.1007/s1 1517-012-0967-8.

[16] A. Ulate-Campos, F. Coughlin, M. Gaínza-Lein, I. S. Fernández, P. L. Pearl, and T. Loddenkemper, "Automated seizure detection systems and their effectiveness for each type of seizure," Seizure, vol. 40, pp. 88-101, 2016/08 2016, doi: 10.1016/j.seizure.2016.06.008.

[17] "EpDetect is a mobile phone application." http://www.epdetect.com (accessed 2021). 
[18] N. A. Marzuki, W. Husain, and A. M. Shahiri, "MyEpiPal: Mobile Application for Managing, Monitoring and Predicting Epilepsy Patient," in Advances in Information and Communication Technology, ed: Springer International Publishing, 2016, pp. 383-392.

[19] R. S. Fisher, E. Bartfeld, and J. A. Cramer, "Use of an online epilepsy diary to characterize repetitive seizures," Epilepsy \& Behavior, vol. 47, pp. 66-71, 2015/06 2015, doi: 10.1016/j.yebeh.2015.04.022.

[20] L. Irody. "Mobile patient diaries: Epdiary." http://www.irody.com/mobile-patient-diaries/ (accessed 2007).

[21] T. Rukasha, S. I. Woolley, and T. Collins, "Wearable epilepsy seizure monitor user interface evaluation," presented at the Adjunct Proceedings of the 2020 ACM International Joint Conference on Pervasive and Ubiquitous Computing and Proceedings of the 2020 ACM International Symposium on Wearable Computers, 2020/09/10, 2020. [Online]. Available: http://dx.doi.org/10.1145/3410530.3414382.

[22] "Empatica Medical-Grade Wearable Patient Monitoring Solutions." http://www.empatica.com/en-eu/ (accessed 2020).

[23] A. Van de Vel et al., "Non-EEG seizure detection systems and potential SUDEP prevention: State of the art," Seizure, vol. 41, pp. 141-153, 2016/10 2016, doi: 10.1016/j.seizure.2016.07.012.

[24] E. Bruno et al., "Wearable technology in epilepsy: The views of patients, caregivers, and healthcare professionals," Epilepsy \& Behavior, vol. 85, pp. 141-149, 2018/08 2018, doi: 10.1016/j.yebeh.2018.05.044.

[25] A. Kos and A. Umek, "Wearable Sensor Devices for Prevention and Rehabilitation in Healthcare: Swimming Exercise With Real-Time Therapist Feedback," IEEE Internet of Things Journal, vol. 6, no. 2, pp. 1331-1341, 2019/04 2019, doi: 10.1109/jiot.2018.2850664.

[26] D. Castaneda, A. Esparza, M. Ghamari, C. Soltanpur, and H. Nazeran, "A review on wearable photoplethysmography sensors and their potential future applications in health care," (in eng), Int J Biosens Bioelectron, vol. 4, no. 4, pp. 195-202, 2018, doi: 10.15406/ijbsbe.2018.04.00125.

[27] P. Jallon, S. Bonnet, M. Antonakios, and R. Guillemaud, "Detection system of motor epileptic seizures through motion analysis with 3D accelerometers," presented at the 2009 Annual International Conference of the IEEE Engineering in Medicine and Biology Society, 2009/09, $2009 . \quad$ [Online]. Available: http://dx.doi.org/10.1109/iembs.2009.5334770.

[28] W. J. C. van Elmpt, T. M. E. Nijsen, P. A. M. Griep, and J. B. A. M. Arends, "A model of heart rate changes to detect seizures in severe epilepsy," Seizure, vol. 15, no. 6, pp. 366-375, 2006/09 2006, doi: 10.1016/j.seizure.2006.03.005.

[29] H. L. Varela, D. S. Taylor, and S. R. Benbadis, "Short-Term Outpatient EEG-Video Monitoring With Induction in a Veterans Administration Population," Journal of Clinical Neurophysiology, vol. 24, no. 5, pp. 390391, 2007/10 2007, doi: 10.1097/wnp.0b013e31812f6c11.

[30] C. Viboud and M. Santillana, "Fitbit-informed influenza forecasts," The Lancet Digital Health, vol. 2, no. 2, pp. e54-e55, 2020/02 2020, doi: $10.1016 / \mathrm{s} 2589-7500(19) 30241-9$.

[31] M. Ntekouli et al., "A mapping of epilepsy's evolution: implementation of the proposed knowledge based model," Evolving Systems, vol. 9, no. 4, pp. 299-313, 2018.

[32] "Focal Onset Seizures (Partial Seizures)." https://www.healthline.com/health/partial-focal-seizure (accessed 2020).

[33] M. Velez, R. S. Fisher, V. Bartlett, and S. Le, "Tracking generalized tonic-clonic seizures with a wrist accelerometer linked to an online database," Seizure, vol. 39, pp. 13-18, 2016.

[34] S. R. Gouravajhala and L. Khuon, "A multi-modality sensor platform approach to detect epileptic seizure activity," in 2012 38th Annual Northeast Bioengineering Conference (NEBEC), 2012: IEEE, pp. 233234.
[35] "EmbracePlus | Empowering Breakthroughs in Neurology Research | Empatica." https://www.empatica.com/embraceplus/ (accessed 2020).

[36] M.-Z. Poh, T. Loddenkemper, N. C. Swenson, S. Goyal, J. R. Madsen, and R. W. Picard, "Continuous monitoring of electrodermal activity during epileptic seizures using a wearable sensor," in 2010 Annual International Conference of the IEEE Engineering in Medicine and Biology, 2010: IEEE, pp. 4415-4418.

[37] "Groundbreaking Announcements as National Epilepsy Awareness Month Begins." https://www.prnewswire.com/newsreleases/groundbreaking-announcements-as-national-epilepsy-awarenessmonth-begins-300176433.html (accessed 2019).

[38] S. A. McHale and E. Pereira, "An IoT Based Epilepsy Monitoring Model," Cham, 2021: Springer International Publishing, in Intelligent Computing, pp. 192-207.

[39] M. Copeland, J. Soh, A. Puca, M. Manning, and D. Gollob, "Microsoft Azure and Cloud Computing," in Microsoft Azure, ed: Apress, 2015, pp. 3-26.

[40] R. K. L. Ko, B. S. Lee, and S. Pearson, "Towards Achieving Accountability, Auditability and Trust in Cloud Computing," in Advances in Computing and Communications, ed: Springer Berlin Heidelberg, 2011, pp. 432-444.

[41] J. M. Peake, G. Kerr, and J. P. Sullivan, "A critical review of consumer wearables, mobile applications, and equipment for providing biofeedback, monitoring stress, and sleep in physically active populations," Frontiers in physiology, vol. 9, p. 743, 2018.

[42] M. Abreu, A. Fred, H. Plácido da Silva, and C. Wang, From Seizure Detection to Prediction: A Review of Wearables and Related Devices Applicable to Epilepsy via Peripheral Measurements. 2020.

[43] "IoT Connectivity Options: Comparing Short-, Long-Range Tech." https://www.iotworldtoday.com/2018/08/19/iot-connectivity-optionscomparing-short-long-range-technologies/ (accessed 2021).

[44] V. Tyndall et al., "Marked improvement in $\mathrm{HbA}(1 \mathrm{c})$ following commencement of flash glucose monitoring in people with type 1 diabetes," (in eng), Diabetologia, vol. 62, no. 8, pp. 1349-1356, 2019, doi: 10.1007/s00125-019-4894-1.

[45] W. P. Institute. "Engineers creating miniaturized, wireless oxygen sensor for sick infants: Mobile, wearable device the size of a Band-Aid could allow babies to leave the hospital and be monitored from home." https://www.sciencedaily.com/releases/2019/11/191114154454.htm (accessed 2019).

[46] F. Porciuncula et al., "Wearable Movement Sensors for Rehabilitation: A Focused Review of Technological and Clinical Advances," (in eng), PM R, vol. 10, no. 9 Suppl 2, pp. S220-S232, 2018, doi: 10.1016/j.pmrj.2018.06.013.

[47] X. Wang, Y. Zhao, and F. Pourpanah, "Recent advances in deep learning," ed: Springer, 2020.

[48] S. Sengupta et al., "A review of deep learning with special emphasis on architectures, applications and recent trends," Knowledge-Based Systems, vol. 194, p. 105596, 2020/04/22/ 2020, doi: https://doi.org/10.1016/j.knosys.2020.105596.

[49] M. K. Siddiqui, R. Morales-Menendez, X. Huang, and N. Hussain, "A review of epileptic seizure detection using machine learning classifiers," (in eng), Brain Inform, vol. 7, no. 1, pp. 5-5, 2020, doi: 10.1186/s40708020-00105-1.

[50] I. Kiral et al., "The Deep Learning Epilepsy Detection Challenge: design, implementation, and test of a new crowd-sourced AI challenge ecosystem," Challenges in Machine Learning Competitions for All (CiML), vol. 1, no. 1, 2019. 